\title{
Holomorphic Besov Spaces on Bounded Symmetric Domains, II
}

\author{
KeHE ZHU
}

\begin{abstract}
The paper continues the study of a class of holomorphic Besov spaces on bounded symmetric domains which was initiated in [18]. Several new descriptions of these Besov spaces are given in terms of weighted Bergman projections and fractional differential operators. These new characterizations are then applied to obtain results about the duality of, and Hankel operators on, weighted Bergman spaces of a bounded symmetric domain.
\end{abstract}

1. Introduction. Let $\Omega$ be an irreducible bounded symmetric domain in $\mathbb{C}^{n}$ in its standard Harish-Chandra realization. A class of holomorphic Besov spaces, denoted $B_{p}(\Omega)(p=0$ or $1 \leq p \leq+\infty)$, are introduced and studied in [18]. The purpose of this paper is to give several more characterizations of the spaces $B_{p}(\Omega)$ and apply them to obtain results about duality of, and Hankel operators on, weighted Bergman spaces on $\Omega$. The remaining part of the introduction is a more specific account of the main results.

Let $d v$ be Lebesgue measure on $\Omega$ normalized so that the total mass of $\Omega$ is 1 . For $0<p<+\infty$ the Bergman space $L_{a}^{p}(\Omega)$ is the closed subspace of $L^{p}(\Omega, d v)$ consisting of holomorphic functions. The Bergman projection $P$ (namely, the orthogonal projection from $L^{2}(\Omega, d v)$ onto $\left.L_{a}^{2}(\Omega)\right)$ is an integral operator:

$$
\operatorname{Pf}(z)=\int_{\Omega} K(z, w) f(w) d v(w), \quad z \in \Omega, f \in L^{2}(\Omega, d v) .
$$

The integral kernel $K(z, w)$ is called the Bergman kernel of $\Omega$.

It is well known [9] that the domain $\Omega$ is uniquely determined (up to a bihomorphic mapping among standard irreducible bounded symmetric domains) by three analytic invariants: $r, a$, and $b$, all of which are nonnegative integers. The invariant $r$ is called the rank of $\Omega$, which is of course always positive. See [9] for the definition of $a$ and $b$; note that the letter $\ell$ is used in [9] for the rank of $\Omega$. We shall make extensive use of the following important invariant of $\Omega$ :

$$
N=a(r-1)+b+2 .
$$


By [6] there exists a polynomial $h(z, w)$ in $z$ and $\bar{w}$ such that the Bergman kernel of $\Omega$ is given by

$$
K(z, w)=\frac{1}{h(z, w)^{N}}, \quad z, w \in \Omega .
$$

The normalizations on $\Omega$ and $d v$ imply that $h(z, 0)=h(0, z)=1$ for all $z \in \Omega$. It is well known that if $\alpha$ is real, then

$$
\int_{\Omega} h(z, z)^{\alpha} d v(z)<+\infty
$$

if and only if $\alpha>-1$.

Throughout this paper we assume $\alpha$ is a real number satisfying $\alpha>-1$. Let $C_{\alpha}$ be a positive normalizing constant such that the measure $d v_{\alpha}(z)=$ $C_{\alpha} h(z, z)^{\alpha} d v(z)$ has total mass 1 on $\Omega$. For $0<p<+\infty$ the weighted Bergman space $L_{a}^{p}\left(d v_{\alpha}\right)$ is the closed subspace of $L^{p}\left(\Omega, d v_{\alpha}\right)$ consisting of holomorphic functions. We let $P_{\alpha}$ denote the orthogonal projection from $L^{2}\left(\Omega, d v_{\alpha}(z)\right)$ onto $L_{a}^{2}\left(d v_{\alpha}\right)$. It can be shown that (see [13] for instance)

$$
P_{\alpha} f(z)=\int_{\Omega} \frac{f(w) d v_{\alpha}(z)}{h(z, w)^{N+\alpha}}, \quad z \in \Omega, f \in L^{2}\left(\Omega, d v_{\alpha}\right) .
$$

Occasionally, we shall write

$$
K_{\alpha}(z, w)=\frac{1}{h(z, w)^{N+\alpha}}, \quad z, w \in \Omega .
$$

We can now state the first main result of the paper.

Theorem A. Suppose $\alpha>-1$ and $1 \leq p \leq+\infty$. Then

$$
\begin{aligned}
& B_{p}(\Omega)=P_{\alpha} L^{p}(\Omega, d \lambda), \\
& B_{0}(\Omega)=P_{\alpha} \mathbb{C}_{0}(\Omega),
\end{aligned}
$$

where $d \lambda(z)=K(z, z) d v(z)$ is the Möbius invariant measure on $\Omega$ and $C_{0}(\Omega)$ is the space of continuous functions on $\Omega$ which vanish on the (topological) boundary $\partial \Omega$ of $\Omega$.

We shall obtain another characterization of $B_{p}(\Omega)$ in terms of a two-parameter family of "fractional differential operators"; see Theorems 4 and 5 in Section 3.

In the rank one case, it is well known that for $1<p<+\infty$ and $p^{-1}+q^{-1}=1$ the dual space of $L_{a}^{p}(\Omega)$ is $L_{a}^{q}(\Omega)$ with the following integral pairing:

$$
\langle f, g\rangle=\int_{\Omega} f(z) \overline{g(z)} d v(z), \quad f \in L_{a}^{p}(\Omega), g \in L_{a}^{q}(\Omega) .
$$

This nice result is not always true on higher rank domains. See [2] for counterexamples. In view of this, our second main result below seems a bit surprising. 
Theorem B. Suppose $0<p \leq 1, \alpha>-1$, and $\beta=(N+\alpha) / p-N$. Then the dual space of $L_{a}^{p}\left(d v_{\alpha}\right)$ can be identified with $B_{\infty}(\Omega)$ under the following integral pairing:

$$
\langle f, g\rangle=\int_{\Omega} f(z) \overline{g(z)} d v_{\beta}(z), \quad f \in L_{a}^{p}\left(d v_{\alpha}\right), \quad g \in B_{\infty}(\Omega) .
$$

Note that the integral above may not be convergent for every $f \in L_{a}^{p}\left(d v_{\alpha}\right)$; it should be understood as a certain limit. The dual space of $L_{a}^{p}\left(d v_{\alpha}\right)$ here is chosen to be independent of $\alpha$ and $p$; the dependence is reflected in the duality pairing. Theorem B was proved in [20] for the open unit ball and in [19] for the open unit polydisk. Earlier work in this area includes [4], [5], [7], [11], and [12].

For a function $f$ in $L_{a}^{2}\left(d v_{\alpha}\right)$ we define an operator

$$
H_{\bar{f}}^{(\alpha)}: L_{a}^{2}\left(d v_{\alpha}\right) \rightarrow L^{2}\left(\Omega, d v_{\alpha}\right)
$$

by

$$
H_{\bar{f}}^{(\alpha)} g=\bar{P}_{\alpha}(\bar{f} g), \quad g \in L_{a}^{2}\left(d v_{\alpha}\right)
$$

where $\bar{P}_{\alpha}$ is the orthogonal projection from $L^{2}\left(\Omega, d v_{\alpha}\right)$ onto the closed subspace

$$
\overline{L_{a}^{2}\left(d v_{\alpha}\right)}=\left\{\bar{f}: f \in L_{a}^{2}\left(d v_{\alpha}\right)\right\} .
$$

The operator $H_{\bar{f}}^{(\alpha)}$ is always densely defined and is called the little Hankel operator on $L_{a}^{2}\left(d v_{\alpha}\right)$.

Theorem C. Suppose $\alpha>-1,1 \leq p<+\infty$, and $f \in L_{a}^{2}\left(d v_{\alpha}\right)$. Then:

(1) The operator $H_{\bar{f}}^{(\alpha)}$ is bounded on $L_{a}^{2}\left(d v_{\alpha}\right)$ if and only if $f \in B_{\infty}(\Omega)$.

(2) The operator $H_{\bar{f}}^{(\alpha)}$ is compact on $L_{a}^{2}\left(d v_{\alpha}\right)$ if and only if $f \in B_{0}(\Omega)$.

(3) The operator $H_{\bar{f}}^{(\alpha)}$ is in the Schatten class $S_{p}$ if and only if $f \in B_{p}(\Omega)$.

The main new ingredient of this theorem is that all characterizations are independent of the parameter $\alpha$. Hankel operators on weighted Bergman spaces of various domains have been studied in a number of papers in recent years; see [8], [16], [17] and references there. Although Theorem C is natural to, and well expected by, experts in this field, its proof in the general case does require a certain amount of work.

2. Preliminaries on differential operators. Let $H(\Omega)$ be the space of all holomorphic functions in $\Omega$. We equip $H(\Omega)$ with the topology of uniform convergence on compact sets. For $f$ in $H(\Omega)$ and $r \in(0,1)$, we define $f_{r} \in H(\Omega)$ by $f_{r}(z)=f(r z), z \in \Omega$; this can be done because $\Omega$ in its standard realization is a convex circular domain in $\mathbb{C}^{n}$. 
Theorem 1. Suppose $\alpha>-1$ and $\beta>-1$. There exists a unique linear operator $D^{\alpha, \beta}: H(\Omega) \rightarrow H(\Omega)$ such that

(1) $D^{\alpha, \beta}$ is continuous on $H(\Omega)$.

(2) $D_{z}^{\alpha, \beta}\left[h(z, w)^{-(N+\beta)}\right]=h(z, w)^{-(N+\beta+\alpha)}$ for all $z, w \in \Omega$.

Proof. This result is proved in [18] in the special case $\beta=0$. The proof for the general case is similar and details will be omitted here. We only want to point out that instead of using the regular Bergman kernel we have to use the following weighted version of the Bergman reproducing formula:

$$
f(z)=C_{\beta} \int_{\Omega} \frac{h(w, w)^{\beta}}{h(z, w)^{N+\beta}} f(w) d v(w),
$$

where $f$ is any function in $L_{a}^{1}\left(d v_{\beta}\right)$.

Just as in the special case $\beta=0$ discussed in [18], the operator $D^{\alpha, \beta}$ admits the following integral representation:

$$
D^{\alpha, \beta} f(z)=C_{\beta} \lim _{r \rightarrow 1^{-}} \int_{\Omega} \frac{h(w, w)^{\beta}}{h(z, w)^{N+\beta+\alpha}} f_{r}(w) d v(w), \quad f \in H(\Omega), z \in \Omega .
$$

In particular, the limit above always exists.

Theorem 2. For $\alpha, \beta>-1$ the operator $D^{\alpha, \beta}$ has the following additional properties:

(1) $D^{\alpha, \beta} f_{r}(z)=D^{\alpha, \beta} f(r z)$ for all $z \in \Omega, r \in(0,1)$, and $f \in H(\Omega)$.

(2) $D^{\alpha, \beta}$ is invertible on $H(\Omega)$ if $\alpha+\beta>-1$.

(3) If $\alpha$ is a positive integer, then $D^{\alpha, \beta}$ is a linear partial differential operator with polynomial coefficients.

Proof. Again the proof of (1) and (2) is similar to the proof in [18] for the special case $\beta=0$. Details are omitted here. Part (3) follows from Theorem 3.17 in [14] and the uniqueness of $D^{\alpha, \beta}$.

Let $D_{\alpha, \beta}$ be the inverse of $D^{\alpha, \beta}$ on $H(\Omega)$. It can be checked that $D_{\alpha, \beta}$ admits the following integral representation:

$$
D_{\alpha, \beta} f(z)=c_{\alpha, \beta} \lim _{r \rightarrow 1^{-}} \int_{\Omega} \frac{h(w, w)^{\alpha+\beta}}{h(z, w)^{N+\beta}} f_{r}(w) d v(w), \quad f \in H(\Omega), z \in \Omega .
$$

Again the limit above always exists.

In the paper [18] we were able to get away with the one-parameter family of operators $D^{\alpha}=D^{\alpha, 0}, \alpha>-1$. But for our later application to the duality problem of weighted Bergman spaces on $\Omega$, we have to make use of the twoparameter family of operators $D^{\alpha, \beta}$, where $\alpha>-1$ and $\beta>-1$. It is not really necessary to restrict $\alpha$ and $\beta$ to the real line; everything will still work if we let $\alpha$ and $\beta$ be complex numbers whose real parts are greater than -1 . We will not need this generality in the paper.

The following technical result will be crucial to our later analysis. 
Theorem 3. Suppose $\alpha>-1$ and $\beta>-1$. Then

$$
D_{z}^{\alpha, \beta}\left[h(z, w)^{-N}\right]=\frac{F(z, w)}{h(z, w)^{N+\alpha}}
$$

and

$$
D_{z}^{N}\left[h(z, w)^{-(N+\beta)}\right]=\frac{G(z, w)}{h(z, w)^{2 N+\beta}},
$$

where $F$ and $G$ are both holomorphic in $z$, conjugate holomorphic in $w$, and bounded in $\Omega \times \Omega$.

Proof. We prove the first identity above; the second one can be proved similarly. From the integral representation of the operator $D^{\alpha, \beta}$ we obtain

$$
D_{z}^{\alpha, \beta}\left[h(z, w)^{-N}\right]=c_{\alpha, \beta} \int_{\Omega} \frac{h(u, u)^{\beta} d v(u)}{h(z, u)^{N+\beta+\alpha} h(u, w)^{N}}, \quad z, w \in \Omega .
$$

Denote the integral above by $f(z, w)$ (forget about the constant). Then $f$ is holomorphic in $z$ and conjugate holomorphic in $w$.

Fix $z \in \Omega$ and let $\varphi_{z}$ be the involutive automorphism interchanging $z$ and 0 ; see [3]. It is well known that the real Jacobian of the mapping $\varphi_{z}(u)$ is given by $h(z, z)^{N} /|h(z, u)|^{2 N}, u \in \Omega$. Thus a change of variables gives

$$
\begin{aligned}
f(z, z) & =\frac{1}{h(z, z)^{N}} \int_{\Omega} \frac{h(u, u)^{\beta}}{h(z, u)^{\beta+\alpha}} \frac{h(z, z)^{N}}{|h(z, u)|^{2 N}} d v(u) \\
& =\frac{1}{h(z, z)^{N}} \int_{\Omega} \frac{h\left(\varphi_{z}(u), \varphi_{z}(u)\right)^{\beta}}{h\left(z, \varphi_{z}(u)\right)^{\beta+\alpha}} d v(u) .
\end{aligned}
$$

It follows from the invariance of the Bergman kernel and the relation $h(z, w)^{-N}=$ $K(z, w)$ that

$$
h(w, z) h\left(\varphi_{z}(w), \varphi_{z}(u)\right) h(z, u)=h(z, z) h(w, u)
$$

for all $z, w, u \in \Omega$. First let $w=0$, and then let $w=u$. We obtain

$$
\begin{aligned}
h\left(z, \varphi_{z}(u)\right) & =\frac{h(z, z)}{h(z, u)}, \quad z, u \in \Omega, \\
h\left(\varphi_{z}(u), \varphi_{z}(u)\right) & =\frac{h(z, z) h(u, u)}{|h(z, u)|^{2}}, \quad z, w \in \Omega .
\end{aligned}
$$

It follows that

$$
f(z, z)=\frac{1}{h(z, z)^{N+\alpha}} \int_{\Omega} \frac{h(z, u)^{\alpha} h(u, u)^{\beta}}{h(u, z)^{\beta}} d v(u) .
$$


Let

$$
F(z, w)=\int_{\Omega} \frac{h(z, u)^{\alpha} h(u, u)^{\beta}}{h(u, w)^{\beta}} d v(u), \quad z, w \in \Omega .
$$

Then $F$ is holomorphic in $z$, conjugate holomorphic in $w$, and

$$
f(z, z)=\frac{F(z, z)}{h(z, z)^{N+\alpha}}
$$

for all $z \in \Omega$. By a well-known uniqueness theorem in several complex variables (see Exercise 3 on page 326 of [10], for example) we must have $f(z, w)=$ $F(z, w) / h(z, w)^{N+\alpha}$ for all $z, w \in \Omega$. It remains to show that $F$ is bounded in $\Omega \times \Omega$.

It is well known that there exists a universal constant $C>0$ such that $h(z, z) \leq C|h(z, w)|$ for all $z$ and $w$ in $\Omega$. If $\beta \geq 0$ and $\alpha \geq 0$, then

$$
|F(z, w)| \leq C^{\beta} \int_{\Omega}|h(z, u)|^{\alpha} d v(u), \quad z, w \in \Omega,
$$

which shows that $F$ is bounded since $h$ is a polynomial. If $\beta \geq 0$ and $-1<\alpha<0$, then

$$
|F(z, w)| \leq C^{\beta+\alpha} \int_{\Omega} h(u, u)^{\alpha} d v(u), \quad z, w \in \Omega,
$$

so that $F$ is bounded. If $-1<\beta<0$ and $\alpha \geq 0$, then the boundedness of $h$ on $\Omega \times \Omega$ implies that there exists a constant $C^{\prime}>0$ such that

$$
|F(z, w)| \leq C^{\prime} \int_{\Omega} h(u, u)^{\beta} d v(u), \quad z, w \in \Omega,
$$

and so $F$ is bounded. Finally, if $-1<\beta<0$ and $-1<\alpha<0$, then the boundedness of $h$ implies that there exists a constant $C^{\prime \prime}>0$ such that

$$
|F(z, w)| \leq C^{\prime \prime} \int_{\Omega}|h(z, u)|^{\alpha} h(u, u)^{\beta} d v(u), \quad z, w \in \Omega .
$$

The boundedness of $F$ in this case follows from a theorem of Faraut and Koranyi; see Theorem 4.1 in [6].

3. Characterizations of Besov spaces. Recall from [18] that the holomorphic Besov space $B_{p}(\Omega), 1 \leq p \leq+\infty$, consists of functions $f$ in $H(\Omega)$ such that $h(z, z)^{N} D^{N} f(z)$ belongs to $L^{p}(\Omega, d \lambda)$, where

$$
d \lambda(z)=h(z, z)^{-N} d v(z)=K(z, z) d v(z)
$$

is the Möbius invariant measure on $\Omega$. The closure of the set of polynomials in $B_{\infty}(\Omega)$ is denoted by $B_{0}(\Omega)$. Equivalently, $B_{0}(\Omega)$ is the space of holomorphic functions $f$ in $\Omega$ such that $h(z, z)^{N} D^{N} f(z)$ belongs to $\mathbb{C}_{0}(\Omega)$. 
Theorem 4. Suppose $1 \leq p<+\infty, \beta>-1$, and

$$
\alpha>\frac{N-1}{p}+\frac{a(r-1)}{2}\left(1-\frac{1}{p}\right) .
$$

Then a holomorphic function $f$ in $\Omega$ belongs to $B_{p}(\Omega)$ if and only if the function $h(z, z)^{\alpha} D^{\alpha, \beta} f(z)$ is in in $L^{p}(\Omega, d \lambda)$.

Proof. First assume $f \in B_{p}(\Omega)$. By Theorem 11 of [18] there exists a function $g$ in $L^{p}(\Omega, d \lambda)$ such that

$$
f(z)=\int_{\Omega} \frac{g(w) d v(w)}{h(z, w)^{N}}, \quad z \in \Omega .
$$

Since the integral above converges uniformly on compact sets and the operator $D^{\alpha, \beta}$ is continuous on $H(\Omega)$, we have

$$
D^{\alpha, \beta} f(z)=\int_{\Omega} D_{z}^{\alpha, \beta}\left[h(z, w)^{-N}\right] g(w) d v(w), \quad z \in \Omega .
$$

By Theorem 3 there exists a constant $C>0$ such that

$$
h(z, z)^{\alpha}\left|D^{\alpha, \beta} f(z)\right| \leq C h(z, z)^{\alpha} \int_{\Omega} \frac{|g(w)| d v(w)}{|h(z, w)|^{N+\alpha}}, \quad z \in \Omega .
$$

It follows from Theorem 9 of [18] (and Lemma 1 there) that the function $h(z, z)^{\alpha} D^{\alpha, \beta} f(z)$ belongs to $L^{p}(\Omega, d \lambda)$.

Next assume that the function $g(z)=h(z, z)^{\alpha} D^{\alpha, \beta} f(z)$ is in $L^{p}(\Omega, d \lambda)$. Recall that $D_{\alpha, \beta}$ is the inverse of $D^{\alpha, \beta}$. We have

$$
f(z)=D_{\alpha, \beta}\left[\frac{g(z)}{h(z, z)^{\alpha}}\right](z)=\int_{\Omega} \frac{h(w, w)^{\beta}}{h(z, w)^{N+\beta}} g(w) d v(w) .
$$

That $g \in L^{p}(\Omega, d \lambda)$ and $\beta>-1$ imply the integral above converges uniformly on compact sets. Thus we can apply the operator $D^{N}$ inside the integral to obtain

$$
D^{N} f(z)=\int_{\Omega} h(w, w)^{\beta} g(w) D_{z}^{N}\left[h(z, w)^{-(N+\beta)}\right] d v(w) . \quad z \in \Omega .
$$

Using Theorem 3 again we obtain a constant $C>0$ such that

$$
h(z, z)^{N}\left|D^{N} f(z)\right| \leq C h(z, z)^{N} \int_{\Omega} \frac{h(w, w)^{\beta}}{|h(z, w)|^{2 N+\beta}}|g(w)| d v(w), \quad z \in \Omega .
$$

By Theorem 9 of [18] the function $h(z, z)^{N} D^{N} f(z)$ is in $L^{p}(\Omega, d \lambda)$. Therefore $f$ belongs to $B_{p}(\Omega)$. 
Theorem 5. Suppose $\beta>-1, \alpha>a(r-1) / 2$, and $f \in H(\Omega)$. Then

(1) $f \in B_{\infty}(\Omega)$ if and only if $h(z, z)^{\alpha} D^{\alpha, \beta} f(z)$ is bounded.

(2) $f \in B_{0}(\Omega)$ if and only if $h(z, z)^{\alpha} D^{\alpha, \beta} f(z)$ is in $\mathbb{C}_{0}(\Omega)$.

Proof. The proof of (1) is similar to that of Theorem 4. To prove (2), first assume $f \in B_{0}(\Omega)$. By Theorem 13 of [18] there exists $g \in \mathbb{C}_{0}(\Omega)$ such that $f=P g$. The proof of Theorem 4 shows that

$$
h(z, z)^{\alpha}\left|D^{\alpha, \beta} f(z)\right| \leq C h(z, z)^{\alpha} \int_{\Omega} \frac{|g(w)| d v(w)}{|h(z, w)|^{N+\alpha}}, \quad z \in \Omega .
$$

If $g$ has compact support in $\Omega$, then the function $h(z, z)^{\alpha} D^{\alpha, \beta} f(z)$ is in $\mathbb{C}_{0}(\Omega)$ since $\alpha>0$ and $h(z, z) \rightarrow 0$ as $z \rightarrow \partial \Omega$. The general case follows from an easy approximation argument.

If the function $g(z)=h(z, z)^{\alpha} D^{\alpha, \beta} f(z)$ is in $\mathbb{C}_{0}(\Omega)$, then by the proof of Theorem 4 we have

$$
h(z, z)^{N}\left|D^{N} f(z)\right| \leq C h(z, z)^{N} \int_{\Omega} \frac{h(w, w)^{\beta}|g(w)| d v(w)}{|h(z, w)|^{2 N+\beta}}, \quad z \in \Omega .
$$

Approximating the function $g$ by continuous functions with compact support in $\Omega$, we conclude that the function $h(z, z)^{N} D^{N} f(z)$ is in $\mathbb{C}_{0}(\Omega)$, and hence $f$ is in $B_{0}(\Omega)$.

Theorem 6. Suppose $1 \leq p \leq+\infty$ and $\beta>-1$. Then $B_{p}(\Omega)=$ $P_{\beta} L^{p}(\Omega, d \lambda)$ and $B_{0}(\Omega)=P_{\beta} \mathbb{C}_{0}(\Omega)$.

Proof. If $f$ is in $B_{p}(\Omega)$, then the function $g(z)=h(z, z)^{N} D^{N, \beta} f(z)$ is in $L^{p}(\Omega, d \lambda)$ by Theorem 4 . Using the integral representation of $P_{\beta}$ we find that $P_{\beta} g=D_{N, \beta} D^{N, \beta} f=f$. This shows that $B_{p}(\Omega) \subset P_{\beta} L^{p}(\Omega, d \lambda)$.

If $f \in P_{\beta} L^{p}(\Omega, d \lambda)$, say,

$$
f(z)=\int_{\Omega} \frac{h(w, w)^{\beta}}{h(z, w)^{N+\beta}} g(w) d v(w),
$$

where $g \in L^{p}(\Omega, d \lambda)$, then by part 2) of Theorem 1

$$
\begin{aligned}
D^{N, \beta} f(z) & =\int_{\Omega} h(w, w)^{\beta} D^{N, \beta}\left[h(z, w)^{-(N+\beta)}\right] g(w) d v(w) \\
& =\int_{\Omega} \frac{h(w, w)^{\beta}}{h(z, w)^{2 N+\beta}} g(w) d v(w) .
\end{aligned}
$$

Applying Theorem 9 of [18] we conclude that the function $h(z, z)^{N} D^{N, \beta} f(z)$ is $L^{p}(\Omega, d \lambda)$, and hence $f$ is in $B_{p}(\Omega)$ by Theorem 4 . This shows that $P_{\beta} L^{p}(\Omega, d \lambda) \subset$ $B_{p}(\Omega)$, and therefore $B_{p}(\Omega)=P_{\beta} L^{p}(\Omega, d \lambda)$.

The proof of the identity $B_{0}(\Omega)=P_{\beta} \mathbb{C}_{0}(\Omega)$ is similar; we omit the details. 
Theorems 4, 5, and 6 are proved in [18] in the special case $\beta=0$. The generalizations here show that "everything" is independent of the new parameter $\beta$. This extra degree of freedom is precisely what we shall need in the next section in dealing with the duality problem for weighted Bergman spaces.

4. Duality of weighted Bergman spaces. Recall that for $0<p<+\infty$ and $\alpha>-1$ the weighted Bergman space $L_{a}^{p}\left(d v_{\alpha}\right)$ is the space of holomorphic functions $f$ in $\Omega$ such that

$$
\|f\|_{\alpha, p}=\left[\int_{\Omega}|f(z)|^{p} d v_{\alpha}(z)\right]^{1 / p}<+\infty .
$$

A linear functional $F$ on $L_{a}^{p}\left(d v_{\alpha}\right)$ is said to be bounded (continuous) if

$$
\|F\|=\sup \left\{|F(f)|:\|f\|_{\alpha, p} \leq 1\right\}<+\infty .
$$

The dual space of $L_{a}^{p}\left(d v_{\alpha}\right)$, denoted $L_{a}^{p}\left(d v_{\alpha}\right)^{*}$, is then the space of all bounded linear functionals on $L_{a}^{p}\left(d v_{\alpha}\right)$. It is easy to see that $L_{a}^{p}\left(d v_{\alpha}\right)^{*}$ is a Banach space for every $p \in(0,+\infty)$ with the norm defined above. In this section we show that each $L_{a}^{p}\left(d v_{\alpha}\right)^{*}, 0<p \leq 1$, can be identified with $B_{\infty}(\Omega)$ via certain volume integral pairing.

Lemma 7. For $\alpha>-1$ and $z \in \Omega$ let

$$
I_{\alpha}(z)=\int_{\Omega} \frac{h(w, w)^{\alpha} d v(w)}{|h(z, w)|^{N+\alpha}} .
$$

Then

$$
\int_{\Omega} I_{\alpha}(z) h(z, z)^{\beta} d v(z)<+\infty
$$

for every $\beta>-1$.

Proof. Recall that $P_{\alpha}$ is the weighted Bergman projection represented by the following integral:

$$
P_{\alpha} f(z)=C_{\alpha} \int_{\Omega} \frac{f(w) h(w, w)^{\alpha} d v(w)}{h(z, w)^{N+\alpha}}, \quad z \in \Omega .
$$

It is clear that

$$
C_{\alpha} I_{\alpha}(z)=\sup \left\{\left|P_{\alpha} f(z)\right|:\|f\|_{\infty} \leq 1\right\}
$$

for every $z \in \Omega$. Fix $\beta>-1$ and let $t$ be a positive number large enough so that $t-\beta>a(r-1) / 2$. Since

$$
P_{\alpha} L^{\infty}(\Omega)=P_{t} L^{\infty}(\Omega)=B_{\infty}(\Omega)
$$


by Theorem 6 , we can find a constant $C>0$ such that

$$
I_{\alpha}(z) \leq C \sup \left\{\left|P_{t} f(z)\right|:\|f\|_{\infty} \leq 1\right\}
$$

for every $z \in \Omega$. For $\|f\|_{\infty} \leq 1$ we have

$$
\left|P_{t} f(z)\right| \leq C_{t} \int_{\Omega} \frac{h(w, w)^{t} d v(w)}{|h(z, w)|^{N+t}} .
$$

It follows from [6] that there exists another constant $C^{\prime}>0$ such that

$$
\begin{aligned}
\int_{\Omega} I_{\alpha}(z) h(z, z)^{\beta} d v(z) & \leq c_{\alpha} C \int_{\Omega} h(z, z)^{\beta} d v(z) \int_{\Omega} \frac{h(w, w)^{t} d v(w)}{|h(z, w)|^{N+t}} \\
& =c_{\alpha} C \int_{\Omega} h(w, w)^{t} d v(w) \int_{\Omega} \frac{h(z, z)^{\beta} d v(z)}{|h(z, w)|^{N+t}} \\
& \leq C^{\prime} \int_{\Omega} \frac{h(w, w)^{t} d v(w)}{h(w, w)^{t-\beta}} \\
& =C^{\prime} \int_{\Omega} h(w, w)^{\beta} d v(w)<+\infty
\end{aligned}
$$

This proves the lemma.

Note that, by using $B_{\infty}(\Omega)=P_{t} L^{\infty}(\Omega)$, we can also show that

$$
\int_{\Omega}|f(z)| h(z, z)^{\beta} d v(z)<+\infty
$$

for every $f$ in $B_{\infty}(\Omega)$ and $\beta>-1$, so that $B_{\infty}(\Omega) \subset L_{a}^{1}\left(d v_{\beta}\right)$ for every $\beta>-1$.

Lemma 8. Suppose $\alpha>-1$. Then

$$
\int_{\Omega} f(z) \overline{g(z)} d v(z)=\int_{\Omega} f(z) \overline{D^{\alpha} g(z)} d v_{\alpha}(z)
$$

for all bounded holomorphic functions $f$ and $g$ in $\Omega$.

Proof. The result follows easily from the integral representation of $D^{\alpha}$, Fubini's Theorem (justified by Lemma 7), and the reproducing property of the operator $P_{\alpha}$. 
Lemma 9. For each $0<p \leq 1$ and $\alpha>-1$ there exists a constant $C>0$ such that

$$
\left.\int_{\Omega}|f(z)| h(z, z)\right|^{(N+\alpha) / p-N} d v(z) \leq C\|f\|_{\alpha, p}
$$

for all $f$ in $L_{a}^{p}\left(d v_{\alpha}\right)$.

Proof. For every $z$ in $\Omega$ let $D(z)$ be the Bergman metric ball with center $z$ and radius 1 . By [1] there exists a constant $C_{1}>0$ such that

$$
|f(z)|^{p} \leq \frac{C_{1}}{v(D(z))} \int_{D(z)}|f(w)|^{p} d v(w)
$$

for all $z \in \Omega, 0<p<+\infty$, and $f \in H(\Omega)$. By Lemmas 6 and 8 in [3] there exists a constant $C_{2}>0$ such that

$$
C_{2}^{-1} \leq \frac{v(D(z))}{h(z, z)^{N}} \leq C_{2}, \quad C_{2}^{-1} \leq \frac{h(z, z)}{h(w, w)} \leq C_{2}
$$

for all $z \in \Omega$ and $w \in D(z)$. This easily implies that there exists a constant $C_{3}>0$ such that

$$
|f(z)| \leq C_{3} h(z, z)^{-(N+\alpha) / p}\|f\|_{\alpha, p}
$$

for all $f \in L_{a}^{p}\left(d v_{\alpha}\right)$ and $z \in \Omega$. Writing $|f(z)|=|f(z)|^{p}|f(z)|^{1-p}$ and applying the estimate above to the second factor, we easily obtain a constant $C>0$ such that

$$
\int_{\Omega}|f(z)| h(z, z)^{(N+\alpha) / p-N} d v(z) \leq C\|f\|_{\alpha, p}
$$

for all $f$ in $L_{a}^{p}\left(d v_{\alpha}\right)$.

The idea for the proof above is due to Dan Luecking. The exponent $(N+$ $\alpha) / p-N$ above is best possible, as can be verified by letting $f$ be large powers of the Bergman kernel.

Theorem 10. Suppose $0<p \leq 1, \alpha>-1$, and $\beta=(N+\alpha) / p-N$. Then we have $L_{a}^{p}\left(d v_{\alpha}\right)^{*}=B_{\infty}(\Omega)$ under the following integral pairing:

$$
\langle f, g\rangle=\lim _{r \rightarrow 1^{-}} \int_{\Omega} f(r z) \overline{g(z)} d v_{\beta}(z), \quad f \in L_{a}^{p}\left(d v_{\alpha}\right), g \in B_{\infty}(\Omega) .
$$

Proof. First, assume $F \in L_{a}^{p}\left(d v_{\alpha}\right)^{*}$. Since $\left\|f_{r}-f\right\|_{\alpha, p} \rightarrow 0$ as $r \rightarrow 1^{-}$for every $f \in L_{a}^{p}\left(d v_{\alpha}\right)$, the continuity of $F$ implies that

$$
F(f)=\lim _{r \rightarrow 1^{-}} F\left(f_{r}\right), \quad f \in L_{a}^{p}\left(d v_{\alpha}\right) .
$$


Since $f_{r}$ is bounded, we have

$$
f_{r}(z)=\int_{\Omega} \frac{f_{r}(w) d v(w)}{h(z, w)^{N}}, \quad z \in \Omega
$$

For every $r \in(0,1)$ the integral above converges in the norm topology of $L_{a}^{p}\left(d v_{\alpha}\right)$. Thus,

$$
F\left(f_{r}\right)=\int_{\Omega} f_{r}(w) F_{z}\left[\frac{1}{h(z, w)^{N}}\right] d v(w)
$$

by the continuity of $F$. Let

$$
\overline{h(w)}=F_{z}\left[\frac{1}{h(z, w)}\right], \quad w \in \Omega
$$

Then $h$ is holomorphic and

$$
F\left(f_{r}\right)=\int_{\Omega} f_{r}(w) \overline{h(w)} d v(w) .
$$

Let $\beta=(N+\alpha) / p-N$. Then $0<p \leq 1$ implies that $\beta \geq \alpha>-1$. Set $g=D^{\beta} h$. We show that $g$ is in $B_{\infty}(\Omega)$. First observe that

$$
\begin{aligned}
\overline{g(w)}=\overline{D^{\beta} h(w)} & =\int_{\Omega} \frac{\overline{h(u)} d v(u)}{h(u, w)^{N+\beta}} \\
& =\int_{\Omega} \frac{d v(u)}{h(u, w)^{N+\beta}} F_{z}\left[\frac{1}{h(z, u)^{N}}\right] \\
& =F_{z} \int_{\Omega} \frac{d v(u)}{h(z, u)^{N} h(u, w)^{N+\beta}} \\
& =F_{z}\left[\frac{1}{h(z, w)^{N+\beta}}\right] .
\end{aligned}
$$

It follows that for every $t>-1$ we have

$$
\begin{aligned}
\overline{D^{t, \beta} g(w)} & =\int_{\Omega} \frac{h(u, u)^{\beta}}{h(u, w)^{N+t+\beta}} F_{z}\left[\frac{1}{h(z, u)^{N+\beta}}\right] d v(u) \\
& =F_{z} \int_{\Omega} \frac{h(u, u)^{\beta} d v(u)}{h(u, w)^{N+t+\beta} h(z, u)^{N+\beta}} \\
& =F_{z}\left[\frac{1}{h(z, w)^{N+t+\beta}}\right] .
\end{aligned}
$$


Since $F$ is bounded on $L_{a}^{p}\left(d v_{\alpha}\right)$, we have

$$
\begin{aligned}
\left|D^{t, \beta} g(w)\right|^{p} & \leq\|F\|^{p} \int_{\Omega} \frac{d v_{\alpha}(z)}{|h(z, w)|^{p(N+t+\beta)}} \\
& =\|F\|^{p} \int_{\Omega} \frac{d v_{\alpha}(z)}{|h(z, w)|^{N+\alpha+p t}} .
\end{aligned}
$$

Choose $t$ large enough so that $t>a(r-1) / 2$ and $\alpha+p t>a(r-1) / 2$. Then by Theorem 4.1 of [6] the function $h(w, w)^{t} D^{t, \beta} g(w)$ is bounded in $\Omega$. This shows that $g$ is in $B_{\infty}(\Omega)$ in view of Theorem 5. In particular, $g \in L^{1}\left(\Omega, d v_{\beta}\right)$. Using Lemma 8 (and a limit argument, namely, $h_{s} \rightarrow h$ as $s \rightarrow 1^{-}$, to justify the use of Lemma 8), we obtain

$$
\begin{aligned}
F\left(f_{r}\right) & =\int_{\Omega} f_{r}(w) \overline{h(w)} d v(w) \\
& =\int_{\Omega} f_{r}(w) \overline{D^{\beta} h(w)} d v_{\beta}(w) \\
& =\int_{\Omega} f_{r}(w) \overline{g(w)} d v_{\beta}(w) .
\end{aligned}
$$

Therefore,

$$
F(f)=\lim _{r \rightarrow 1^{-}} \int_{\Omega} f(r z) \overline{g(z)} d v_{\beta}(z), \quad f \in L_{a}^{p}\left(d v_{\alpha}\right), g \in B_{\infty}(\Omega) .
$$

This shows that every bounded linear functional on $L_{a}^{p}\left(d v_{\alpha}\right)$ arises from a function in $B_{\infty}(\Omega)$ under the integral pairing with measure $d v_{\beta}$.

Next, assume that $g$ is a function in $B_{\infty}(\Omega)$. We show that $g$ gives rise to a bounded linear functional on $L_{a}^{p}\left(d v_{\alpha}\right)$ under the same integral pairing. By Theorem 6 there exists a bounded function $\varphi$ on $\Omega$ such that

$$
g(z)=P_{\beta} \varphi(z)=\int_{\Omega} \frac{\varphi(w) d v_{\beta}(w)}{h(z, w)^{N+\beta}}, \quad z \in \Omega .
$$

Using Fubini's Theorem (whose use is justified by Lemma 7) and the reproducing property of $P_{\beta}$, we easily obtain

$$
\int_{\Omega} f_{r}(z) \overline{g(z)} d v_{\beta}(z)=\int_{\Omega} f_{r}(w) \overline{\varphi(w)} d v_{\beta}(w), \quad r \in(0,1) .
$$

Since $L_{a}^{p}\left(d v_{\alpha}\right) \subset L_{a}^{1}\left(d v_{\beta}\right)$ by Lemma 9 , we conclude that

$$
\lim _{r \rightarrow 1^{-}} \int_{\Omega} f_{r}(z) \overline{g(z)} d v_{\beta}(z)=\int_{\Omega} f(z) \overline{\varphi(z)} d v_{\beta}(z), \quad f \in L_{a}^{p}\left(d v_{\alpha}\right),
$$

and this formula defines a bounded linear functional on $L_{a}^{p}\left(d v_{\alpha}\right)$ with norm less than or equal to $C\|\varphi\|_{\infty}$, where $C$ is the constant from Lemma 9 . This completes the proof of Theorem 10. 
Note that Theorem 10 was proved in [15], [16] in the case $p=1$ and $\beta=0$. Using methods developed in the present paper, we can easily prove that for every $\beta>-1$ the dual space of $B_{0}(\Omega)$ can be identified with $L_{a}^{1}\left(d v_{\beta}\right)$ under the integral pairing with measure $d v_{\beta}$.

Using the term "Mackey topology" (see [12]), we can rephrase Theorem 10 as follows.

Corollary 11. Suppose $\alpha>-1,0<p \leq 1$, and $\beta=(N+\alpha) / p-N$. Then the norm of $L_{a}^{1}\left(d v_{\beta}\right)$ induces the Mackey topology on $L_{a}^{p}\left(d v_{\alpha}\right)$; and $L_{a}^{1}\left(d v_{\beta}\right)$ is the Mackey completion of $L_{a}^{p}\left(d v_{\alpha}\right)$.

We have been unable to identify the dual space of the Hardy space $H^{p}(\Omega)$ for $0<p<1$. The main difficulty here is the lack of a sharp Hardy-Littlewood type inequality for embedding Hardy spaces $H^{p}(\Omega), 0<p<1$, into weighted Bergman $L^{1}$ spaces. Theorem 11 in [11] is a Hardy-Littlewood type result, but we do not think it is sharp enough.

5. Hankel operators on weighted Bergman spaces. Recall that for $\alpha>-1$ the orthogonal projection from $L^{2}\left(\Omega, d v_{\alpha}\right)$ onto the closed subspace of conjugate holomorphic functions is denoted by $\bar{P}_{\alpha}$. For $\varphi \in L^{2}\left(\Omega, d v_{\alpha}\right)$ the little Hankel operator $H_{\varphi}^{(\alpha)}: L_{a}^{2}\left(d v_{\alpha}\right) \rightarrow L^{2}\left(\Omega, d v_{\alpha}\right)$ is defined by $H_{\varphi}^{(\alpha)} f=\bar{P}_{\alpha}(\varphi f), f \in$ $L_{a}^{2}\left(d v_{\alpha}\right)$. Although the operator $H_{\varphi}^{(\alpha)}$ is not always bounded, it is always densely defined.

Theorem 12. Suppose $f \in L_{a}^{2}\left(d v_{\alpha}\right)$ and $1 \leq p<+\infty$. Then:

(1) $H_{\bar{f}}^{(\alpha)}$ is bounded on $L_{a}^{2}\left(d v_{\alpha}\right)$ if and only if $f \in B_{\infty}(\Omega)$.

(2) $H_{\bar{f}}^{(\alpha)}$ is compact on $L_{a}^{2}\left(d v_{\alpha}\right)$ if and only if $f \in B_{0}(\Omega)$.

(3) $H_{\bar{f}}^{(\alpha)}$ is in the Schatten class $S_{p}$ if and only if $f \in B_{p}(\Omega)$.

Proof. This follows from Corollary 24 in [18] and Theorem 6 in Section 3.

Note that the Hankel operators we considered here are usually called little Hankel operators. Given $\varphi \in L^{2}\left(\Omega, d v_{\alpha}\right)$, we can also consider the operator on $L_{a}^{2}\left(d v_{\alpha}\right)$ defined as follows:

$$
f \mapsto\left(I-P_{\alpha}\right)(\varphi f), \quad f \in L_{a}^{2}\left(d v_{\alpha}\right) .
$$

Such operators are usually called big Hankel operators. When the rank of $\Omega$ is greater than one, there exist major differences between size estimates for little Hankel operators and those for big Hankel operators. See [1]. 


\section{REFERENCES}

[1] D. Bekolle, C. Berger, L. Coburn, And K. Zhu, BMO in the Bergman metric on bounded symmetric domains, J. Funct. Anal. 91 (1990), 310-350.

[2] D. Bekolle And A. Bonami, Estimates for the Bergman and Szegö projections in two symmetric domains of $\mathbb{C}^{n}$, Colloq. Math. 68 (1995), 81-100.

[3] C. Berger, L. Coburn, And K. Zhu, Function theory on Cartan domains and the Berezin-Toeplitz symbol calculus, Amer. J. Math. 110 (1988), 921-953.

[4] R. Coifman And R. Rochberg, Representation theorems for holomorphic and harmonic functions in $L^{p}$, Astérisque 77 (1980), 11-66.

[5] P. Duren, B. Romberg, And A. Shields, Linear functionals on $H^{p}$ spaces with $0<p<1$, J. Reine Angew. Math.vol 238 (1969), 32-60.

[6] J. FARAUt AND A. KorAnyI, Function spaces and reproducing kernels on bounded symmetric domains, J. Funct. Anal. 88 (1990), 64-89.

[7] A. Frazier, The dual of $H^{p}$ of the polydisk for $0<p<1$, Duke Math. J. 39 (1972), 369-379.

[8] S. Janson, J. Peetre, And R. RochBerg, Hankel forms and the Fock space, Revista Mat. Ibero-Amer. 3 (1987), 61-138.

[9] A. Koranyi, Analytic invariants of bounded symmetric domains, Proc. Amer. Math. Soc. 19 (1968), 279-284.

[10] S. KrantZ, Function Theory of Several Complex Variables, John Wiley \& Sons, 1982.

[11] J. Mitchell AND K. HAhn, Representation of linear functionals in $H^{p}$ spaces over bounded symmetric domains in $\mathbb{C}^{n}$, J. Math. Anal. Appl. 56 (1976), 379-396.

[12] J. ShAPIRO, Mackey topologies, reproducing kernels, and diagonal maps on the Hardy and Bergman spaces, Duke Math. J. 43 (1976), 187-202.

[13] M. Stoll, Mean value theorems for harmonic and holomorphic functions on bounded symmetric domains, J. Reine Angew. Math. 283 (1977), 191-198.

[14] Z. YAN, Invariant differential operators and holomorphic function spaces, preprint.

[15] Z. YAN, Duality and differential operators on the Bergman spaces of bounded symmetric domains, J. Funct. Anal. 105 (1992), 171-186.

[16] K. ZHU, Duality and Hankel operators on the Bergman spaces of bounded symmetric domains, J. Funct. Anal. 81 (1988), 260-278.

[17] K. ZHU, Hankel operators on the Bergman spaces of bounded symmetric domains, Trans. Amer. Math. 324 (1991), 707-730.

[18] K. ZHU, Holomorphic Besov spaces on bounded symmetric domains, Quarterly J. Math. Oxford (2) 46 (1995), 239-256.

[19] K. ZHU, Weighted Bergman projections on the polydisk, Houston J. Math. 20 (1994), 275-292.

[20] K. ZHU, Bergman and Hardy spaces with small exponents, Pacific J. Math. vol 162 (1994), 189-199.

Research supported by the National Science Foundation.

Department of Mathematics and Statistics

State University of New York at Albany

Albany, NY 12222, USA

Received: January 21st, 1994; revised: July 6th, 1994. 